\title{
JPEG Image Transmission over Rayleigh Fading Channel with Unequal Error Protection
}

\author{
J. N. Patel \\ Phd,Assistant Professor, \\ ECED \\ SVNIT, Surat
}

\author{
S. Patnaik \\ Phd,Professor, ECED \\ SVNIT, Surat
}

\author{
Murali. D \\ PG Student, ECED \\ SVNIT, Surat
}

\begin{abstract}
In this paper an image transmission system has been proposed where Joint Photographic Experts Group (JPEG) algorithm is used as an image coder and Rate Compatible Punctured Convolution (RCPC) channel coder is used for transmission of coded image over wireless channels (AWGN and Rayleigh fading). JPEG bit stream is partitioned into DC and AC bit streams. AC bit stream further classified using edge density property of block. Priorities based Unequal error Protection (UEP) applied to bit stream. Distortion analysis (MSE) is given for proposed image transmission scheme. The simulation results shows reduction in distortion compared to conventional Equal Error Protection (EEP). This proposed algorithm can be applied for low frequency as well as high frequency images.
\end{abstract}

\section{General Terms}

Image Coding and transmission through wireless channel

\section{Keywords}

Joint Photographic Experts Group (JPEG), Rate Compatible Punctured Convolutional (RCPC), Mean Square Error (MSE), Equal Error Protection (EEP), Unequal Error Protection (UEP).

\section{INTRODUCTION}

Wireless channel characteristics like fading, Inter Symbol Interference (ISI) prohibit the reliable transmission of uncompressed image. As there are constraints on bandwidth, power etc, there is always trade-off between source coding and channel coding. Shanon has given separate source channel coding transmission system [1]. Using Joint Source Channel Coding (JSCC) recent wireless communication technology lead to robust and reliable image transmission [2] [3].The basic block diagram consists of source encoder/decoder and channel encoder/decoder as shown in Figure 1. Source encoder is used to reduce the amount of data necessary to represent the information of the Image signal. The objective of the channel encoder is to add redundancy to the output of the source encoder to enhance the reliability on the transmission.

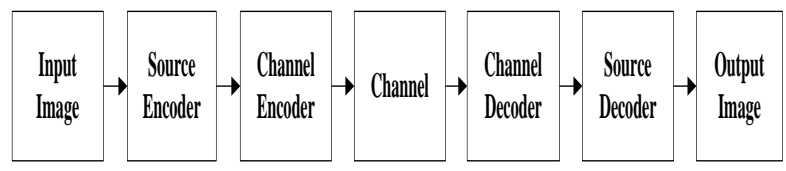

Fig 1: Image Transmission System

Due to wireless channel characteristics, Variable length coding at encoding stage received image quality degrades. So to combat channel errors bit stream has applied protection using different channel code. This is Equal Error Protection (EEP) algorithm. But as in multimedia signal like image, audio, video whole bit stream importance in received signal is not same. So Bitstream is partitioned and protection assigned according to importance. This concept defines Unequal Error Protection (UEP). The various image coding algorithms with UEP transmission is mentioned in [4][5][6][7]. In this paper EEP, UEP and UEP_ED algorithms implemented and their simulation results compared. In UEP JPEG stream classified as AC bit stream and DC bit stream where as in UEP_ED algorithm AC bit stream is further classified.

This paper is organised as follows. Section 2 gives detail of source code algorithm JPEG with simulation results. Section 3 gives Bit Error Rate (BER) performance of RCPC channel codec, Section 4 describes in detail UEP_ED algorithm with simulation results and comparison of EEP, UEP and UEP_ED. Section 5 conclude the algorithm.

\section{THE IMAGE COMPRESSION STANDARD: JPEG}

The original goal of JPEG is to provide still Image compression techniques for a large range of types of images [8] by exploiting redundancy in the signal. The Discrete Cosine Transform (DCT) gives DC coefficient and AC coefficients. The quantization step is responsible for the source distortion in the codec and determines the compression. This quantization step size can be varied using Quality Factor (QF). The QF will decide the source coding rate Rs (Bits per pixel, Bpp) The quantized DC and AC coefficients found using following equation.

Quantized coefficient $(i, j)=\operatorname{round}\left(\frac{D C T(i, j)}{Q(i, j)}\right)$

DC coefficients further process by Differential Pulse Code Modulation (DPCM) and Huffman coding whereas AC coefficients further process using Run Length Coding (RLC) and Huffman coding [8]. According to JPEG algorithm the source rate distortion curve is shown in Figure 2(a) for Barbara test image. Corresponding compression ratio also mentioned in Figure 2(b). At very high compression, decoded image perceptual quality suffers from blocking artifacts. The performance measurement parameter Mean Square Error (MSE) is calculated for M X N size image is given by:

$$
M S E=\frac{\sum_{x=1}^{M} \sum_{y=1}^{N}[I(x, y)-\hat{l}(x, y)]^{2}}{M * N}
$$

Where $I(x, y)$ is the original image, $\hat{I}(x, y)$ is reconstructed image. The Peak Signal to Noise Ratio (PSNR) is calculated from the obtained MSE.

$$
P S N R=10 \log _{10}\left[\frac{\left(2^{n}-1\right)^{2}}{M S E}\right]
$$




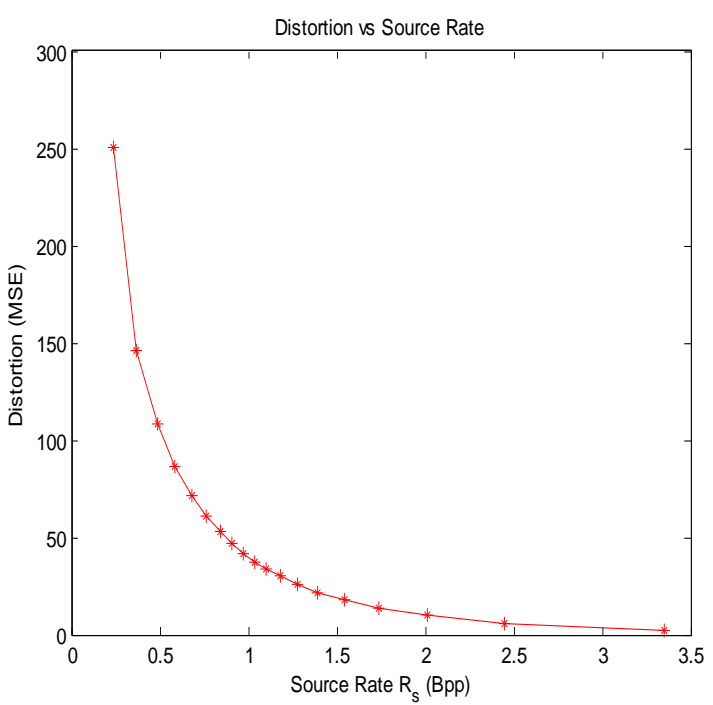

(a)

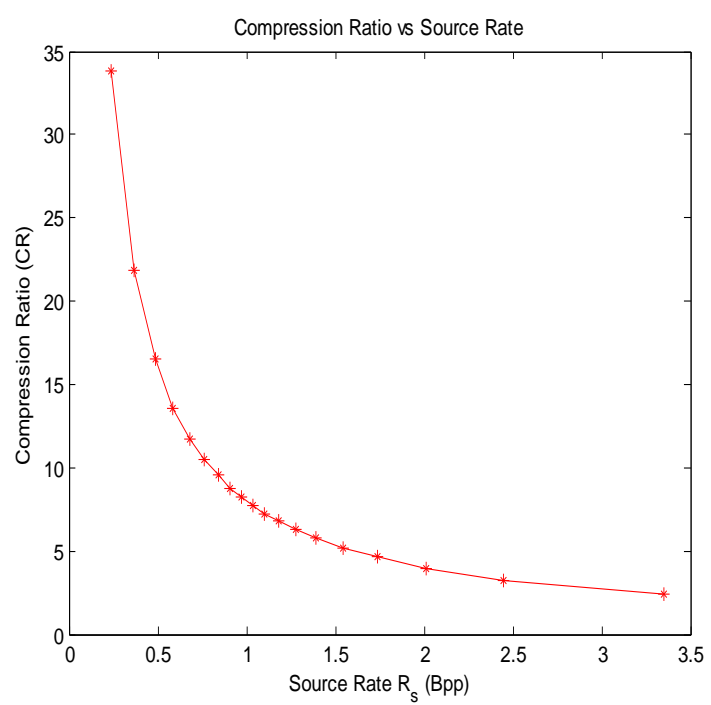

(b)

Fig 2: (a) Source Rate $\mathbf{R}_{\mathrm{s}}(\mathrm{Bpp})$ vs Distortion (MSE) curve for Barbra image (b) Source Rate $\mathbf{R}_{\mathrm{s}}$ (Bpp) vs Compression Ratio (CR).

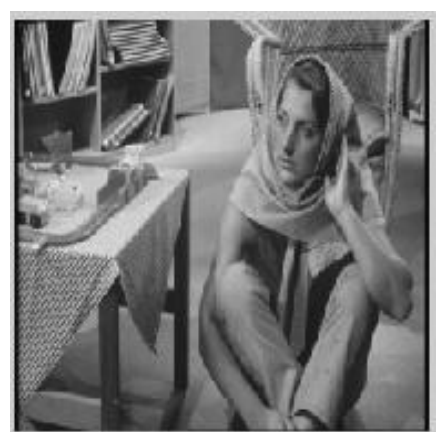

(a)

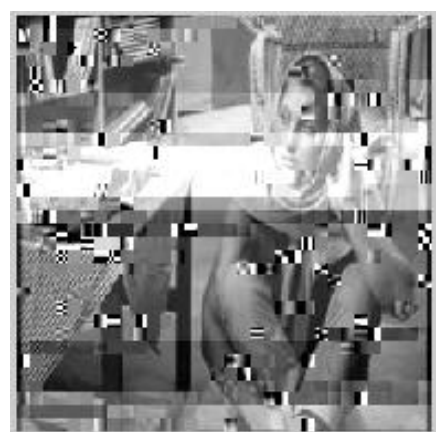

(b)

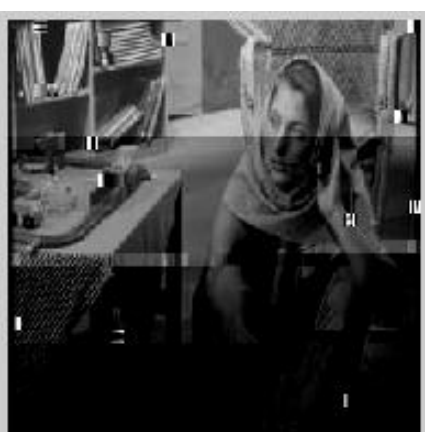

(c)

Fig 3: (a) Original Barbara Image. (b), (c) Received image from Rayleigh channel at $\mathrm{SNR}=20 \mathrm{~dB}, \mathrm{SNR}=25 \mathrm{~dB}$

The compressed bit stream is modulated (BPSK modulation) and passed through Rayleigh fading channel. The last stage of JPEG encoder use Variable Length Coding (VLC) which create the synchronization problem at the decoder. The simulations assume ideal synchronization. The following Figure 3 shows the effect of channel noise on decoded image. In this case the total end to end distortion is source distortion $\left(D_{\mathrm{s}}\right)$ as well as channel distortion $\left(\mathrm{D}_{\mathrm{c}}\right)$.

$$
D_{\text {Total }}=D_{s}+D_{c}
$$

To minimize the impact of transmission error, an appropriate choice of channel error correcting and detecting codes is necessary, Error resilient technology and error concealment technology can be applied to obtain better perceptual quality.

\section{CHANNEL CODER: RCPC}

The Rate Compatible Punctured Convolution Coder (RCPC) is used as Forward Error Correcting (FEC) code. This code is defined in Hagenauer [9] with its application. The convolution coder of mother code rate $\mathrm{R}=1 / \mathrm{N}=1 / 3$ with

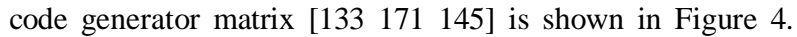
Generally a rate $(\mathrm{P} / \mathrm{P}+\mathrm{I})$ punctured convolution code can be obtained by periodically puncturing a low rate mother code rate $1 / \mathrm{N}$ with period $\mathrm{P}(\mathrm{P}=8$ in this simulation $)$ and $1 \leq \mathrm{I} \leq(\mathrm{N}$ 1) $P$. So different channel code rate $R_{c}=8 / 9,8 / 10 \ldots 8 / 24$ are generated. The basic procedure for constructing high rate punctured code from low rate $1 / \mathrm{N}$ is mother code followed by puncturing procedure. This delete the encoded output symbols using a puncturing matrix $\mathrm{P}$ (i) with size N X P. One of the examples with all the detail steps mentioned below.

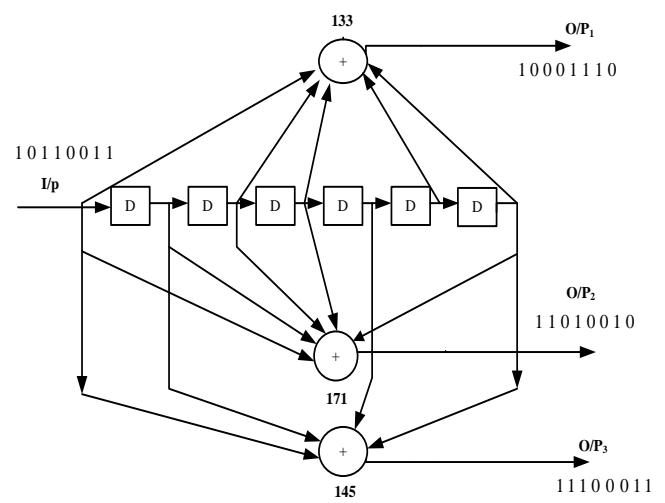

Fig 4: Convolution coder of $R=1 / N=1 / 3$ Mother code rate 
Example of RCPC Coder:

Puncturing period $\mathrm{P}=8$, puncturing matrix size $\mathrm{P}(\mathrm{i})=\mathrm{N} \mathrm{X} \mathrm{p}=3 \mathrm{X} 8$

$$
\begin{aligned}
& P_{8 / 12}=\left[\begin{array}{llllllll}
1 & 1 & 1 & 1 & 1 & 1 & 1 & 1 \\
1 & 0 & 1 & 0 & 1 & 0 & 1 & 0 \\
0 & 0 & 0 & 0 & 0 & 0 & 0 & 0
\end{array}\right] \\
& P_{8 / 16}=\left[\begin{array}{llllllll}
1 & 1 & 1 & 1 & 1 & 1 & 1 & 1 \\
1 & 1 & 1 & 1 & 1 & 1 & 1 & 1 \\
0 & 0 & 0 & 0 & 0 & 0 & 0 & 0
\end{array}\right]
\end{aligned}
$$

Input bitstream

$:\left[\begin{array}{llllllll}1 & 0 & 1 & 1 & 0 & 0 & 1 & 1\end{array}\right]$

Mother convolutional coder

$\mathrm{O} / \mathrm{P}_{1}:\left[\begin{array}{llllllll}1 & 0 & 0 & 0 & 1 & 1 & 1 & 0\end{array}\right]$

$\mathrm{O} / \mathrm{P}_{2}:\left[\begin{array}{llllllll}1 & 1 & 0 & 1 & 0 & 0 & 1 & 0\end{array}\right]$

$\mathrm{O} / \mathrm{P}_{3}:\left[\begin{array}{llllllll}1 & 1 & 1 & 0 & 0 & 0 & 1 & 1\end{array}\right]$

So the output of the $1 / 3$ convolutional coder :

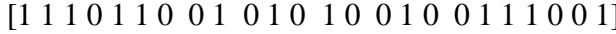

After applying puncturing matrix $\mathrm{P}_{8 / 12}$ :

$\left[\begin{array}{llllllllllll}1 & 1 & 0 & 0 & 0 & 0 & 1 & 0 & 1 & 1 & 1 & 0\end{array}\right]$

After applying puncturing matrix $\mathrm{P}_{8 / 16}$ :

$\left[\begin{array}{lllllllllllllll}1 & 1 & 0 & 1 & 0 & 0 & 0 & 1 & 1 & 0 & 1 & 0 & 1 & 1 & 0\end{array}\right]$

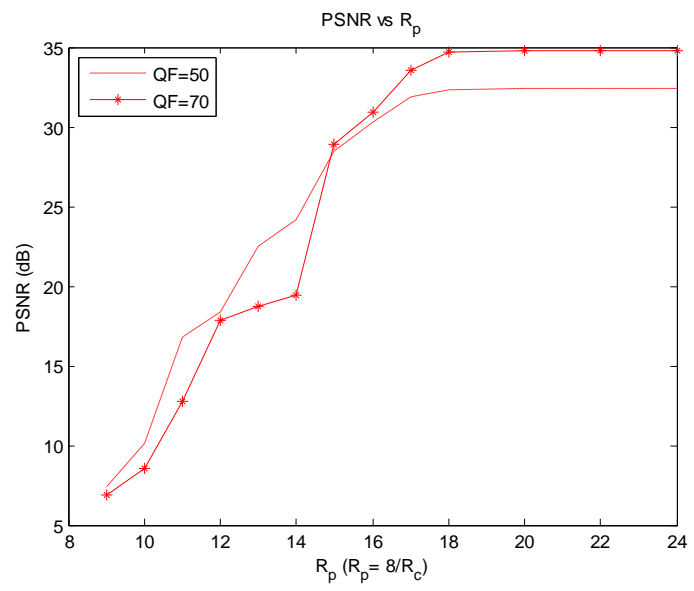

Fig 5: $R_{p}$ vs PSNR at $S N R=10 d B$

The Bit Error Rate (BER) performance for different SNR is shown in Table 1. Table 1 suggests the possible RCPC code rate for a given SNR and desired BER. For SNR 10dB, the BER value 0.005 can be possible with $R_{c}=8 / 12$.

For SNR 10dB, $R_{p}$ versus PSNR curve is shown in Figure 5 where $R_{p}=8 / R_{c}$ for $Q F=50$ and $Q F=70$. It is observed that up to channel code rate $R_{c}=8 / 18$ (corresponding $R_{p}=18$ ) channel error affect the received quality. For further $R_{p}$ rate all the transmitted encoded stream have zero channel error. So in received image only source distortion $\left(D_{s}\right)$ effect remains present. The PSNR value remains constant after $R_{p}=18$ as BER value becomes zero for further rate.

\section{IMAGE TRANSMISSION OVER CHANNEL}

When encoded image stream has applied same protection level it is defined as Equal Error Protection (EEP). In Unequal Error Protection (UEP) data can be partitioned based on importance of pixels for decoding the image. JPEG encoded stream has DC coefficient bit stream and AC coefficient bit stream. DC coefficients carry average intensity value of $8 \mathrm{X} 8$ blocks. The impact of error in $\mathrm{AC}$ coefficients $\left[\mathrm{P}_{\mathrm{e}(\mathrm{AC})}\right]$ and in $\mathrm{DC}$ coefficients $\left[\mathrm{P}_{\mathrm{e}(\mathrm{DC})}\right]$ on decoded image can be visualised in Figure 6. Figure 6 a), b), c) are the received images corresponding to decreasing error of AC coefficient bitstream $\mathrm{P}_{\mathrm{e}(\mathrm{AC})}$ while maintaining DC coefficient bitstream error $P_{e(D C)}=0$. These errors are limited to respected blocks only. The Figure $6 \mathrm{~d}$ ), e) and f) shows the error in DC coefficient bitstream with maintaining $\mathrm{AC}$ coefficient error $\mathrm{P}_{\mathrm{e}(\mathrm{AC})}$. This error propagates and changes the intensity level of blocks. The symbol $R_{c}^{D C}$ and $R_{c}^{A C}$ indicates channel code rate for DC stream and $\mathrm{AC}$ stream. It is concluded that if error in DC coefficients is less than improvement in received image will be higher compared to vice versa case of AC coefficients. AC coefficients error will effect detail information of image. So DC coefficients should provide higher protection compared to AC coefficients. In UEP algorithm data partioned in two groups DC coefficient stream and AC coefficient stream. Further AC coefficients stream will be partitioned based on the each block property edge density. Using this property, AC coefficients of blocks classified in one of category as high edge density block coefficients bitstream or low edge density bit stream.

So finally JPEG Encoded Bit stream partitioned into three groups: DC coefficients, High Edge Density AC coefficients, Low Edge Density AC coefficients in UEP_ED algorithm. Edge Density calculation can be applied on each 8 X8 blocks as following. Consider

$c_{i} \quad=$ Number of ones in $\mathrm{i}^{\text {th }}$ block after applying Sobel operator to each block

$c_{i e}=c_{i} / 64=$ edge density of block

$B l k_{1}=\left[c_{1}, c_{2}, c_{3}, \ldots \ldots . c_{i}\right]$

$\mathrm{i}=$ block number.

Edge density of an image,

$$
B l k_{1 m}=\frac{C_{1}+C_{2}+C_{3}+\cdots \ldots+C_{i}}{\text { Total Blocks }}
$$

If $c_{i e} \geq B l k_{1 m} \quad$ Block is classified as HIGH edge density blocks, and marked for higher protection

If $c_{i e}<B l k_{1 m} \quad$ Block is classified as LOW edge density blocks, and marked for lower protection

Table 1. Bit Error Rate performance of RCPC for Rayleigh fading channel

\begin{tabular}{|c|c|c|c|c|c|c|c|}
\hline $\begin{array}{l}\text { SNR(dB) } \\
\text { Channel } \\
\text { Rate }\end{array}$ & $\mathbf{0}$ & $\mathbf{5}$ & $\mathbf{1 0}$ & $\mathbf{1 5}$ & $\mathbf{2 0}$ & $\mathbf{2 5}$ \\
\cline { 2 - 8 } & \multicolumn{7}{|c|}{ BER } \\
\hline $8 / 9$ & 0.485 & 0.421 & 0.151 & 0.026 & 0.002 & $7.64 \mathrm{E}-4$ \\
\hline $8 / 10$ & 0.472 & 0.318 & 0.052 & 0.003 & $3.96 \mathrm{E}-4$ & $1.61 \mathrm{E}-4$ \\
\hline $8 / 12$ & 0.433 & 0.125 & 0.005 & $8.81 \mathrm{E}-5$ & 0 & 0 \\
\hline $8 / 16$ & 0.265 & 0.015 & $1.616 \mathrm{E}-4$ & 0 & 0 & 0 \\
\hline $8 / 20$ & 0.124 & 0.001 & 0 & 0 & 0 & 0 \\
\hline
\end{tabular}

$R_{c}^{D C}=8 / 16, R_{c}^{A C}=8 / 10$

$R_{c}^{D C}=8 / 16, R_{c}^{A C}=8 / 12$

$R_{c}^{D C}=8 / 16, R_{c}^{A C}=8 / 14$ 


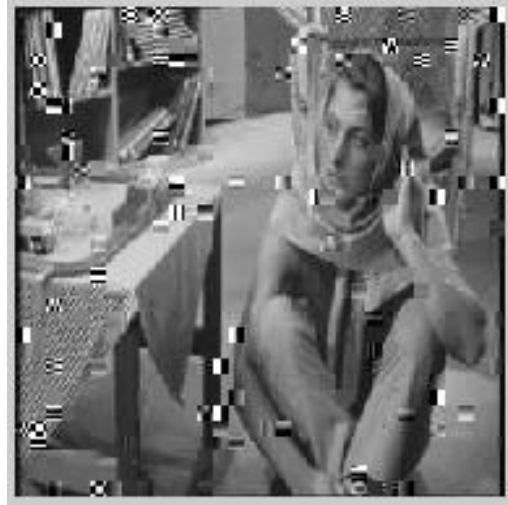

$\mathrm{P}_{\mathrm{e}(\mathrm{DC})}=0, \mathrm{P}_{\mathrm{e}(\mathrm{AC})}=0.0502$

$\mathrm{MSE}=817.573, \mathrm{PSNR}=19.006$

(a)

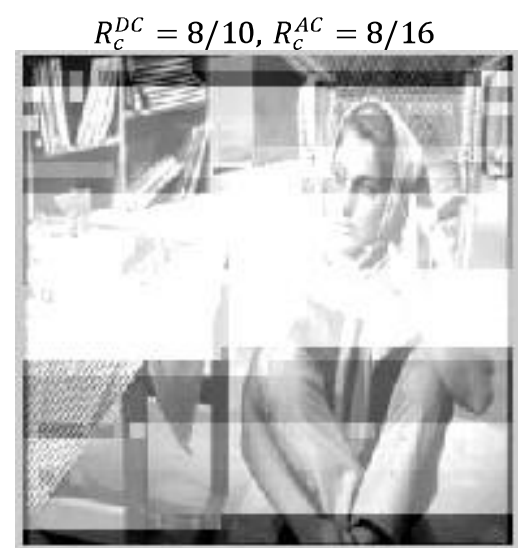

$\mathrm{P}_{\mathrm{e}(\mathrm{DC})}=0.0369, \mathrm{P}_{\mathrm{e}(\mathrm{AC})}=2.44 \mathrm{E}-4$ $\mathrm{MSE}=9.174 \mathrm{E}+3, \mathrm{PSNR}=8.505$

(d)

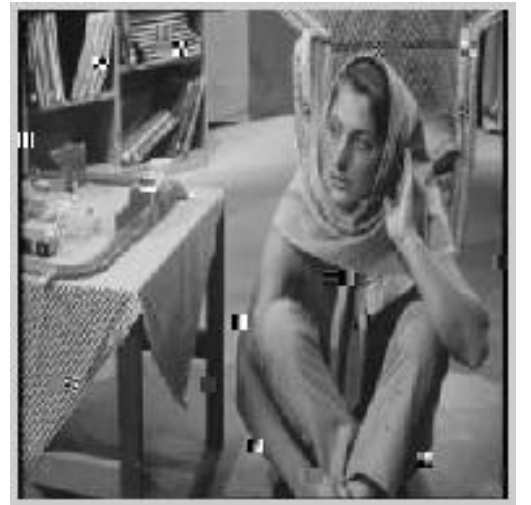

$\mathrm{P}_{\mathrm{e}(\mathrm{DC})}=0, \mathrm{P}_{\mathrm{e}(\mathrm{AC})}=0.0047$

$\mathrm{MSE}=162.240, \mathrm{PSNR}=26.029$

(b)

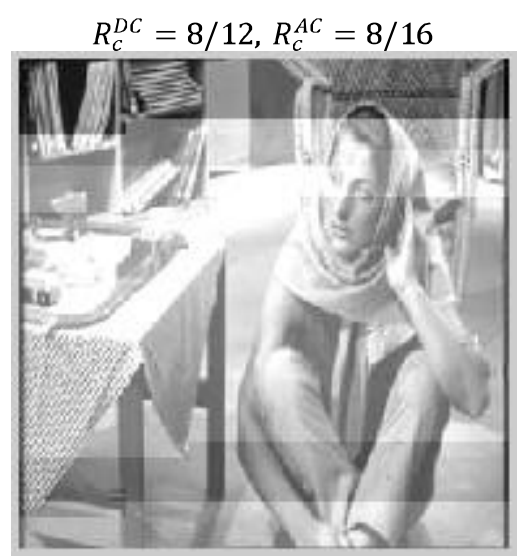

$\mathrm{P}_{\mathrm{e}(\mathrm{DC})}=0.0042, \mathrm{P}_{\mathrm{e}(\mathrm{AC})}=2.44 \mathrm{E}-4$

$\mathrm{MSE}=5.207 \mathrm{E}+3, \mathrm{PSNR}=10.965$

(e)

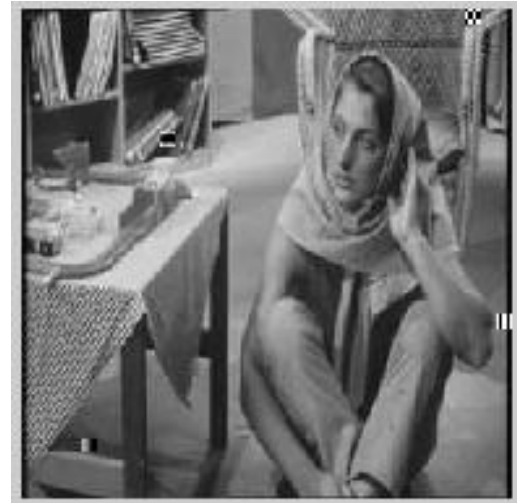

$\mathrm{P}_{\mathrm{e}(\mathrm{DC})}=0, \mathrm{P}_{\mathrm{e}(\mathrm{AC})}=8.146 \mathrm{E}-4$ $\mathrm{MSE}=85.355, \mathrm{PSNR}=28.818$

(c)

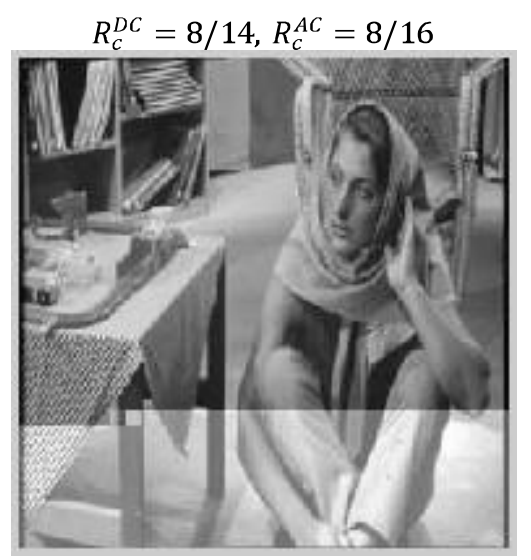

$\mathrm{P}_{\mathrm{e}(\mathrm{DC})}=0.0015, \mathrm{P}_{\mathrm{e}(\mathrm{AC})}=2.44 \mathrm{E}-4$ $\mathrm{MSE}=1.99 \mathrm{E}+3, \mathrm{PSNR}=15.137$

(f)

Fig 6: (a) (b) (c) Error in only AC coefficients, (d) (e) (f) Error in only DC coefficients.

The threshold value 0.1 is used for sobel operator. If this value reduces false edges also starts detected. Final system block diagram for this system is shown in Figure 7. The bit stream mixer finally transmitted bit stream of $\mathrm{R}_{\text {Total }}$, that is equal to $R_{c}^{D C}$ followed by $R_{c-H}^{A C}$ and $R_{c-L}^{A C}$ The symbol $R_{c-H}^{A C}$ is defined channel code rate for high edge density AC bitstream and $R_{C-L}^{A C}$ is defined for low edge density $\mathrm{AC}$ bit stream.

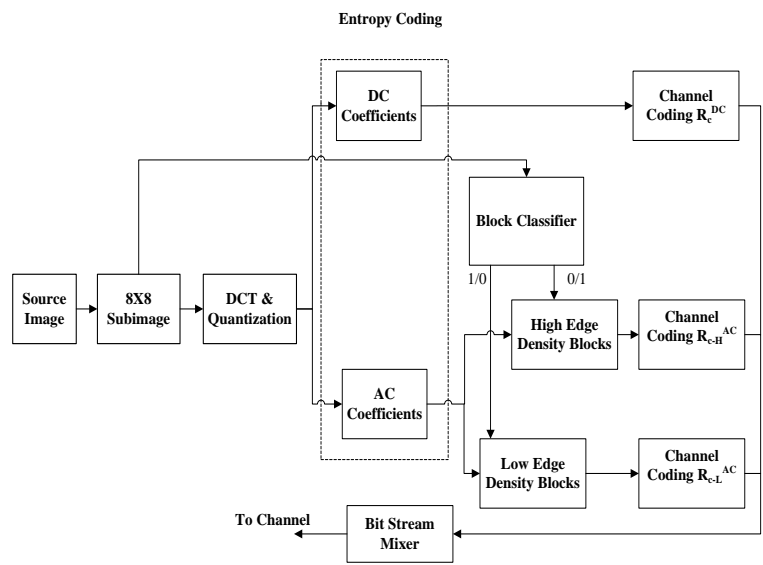

Fig 7: Block Diagram of UEP_ED

\section{SIMULATION RESULTS}

The simulation result is carried for fixed total transmission rate $\left(\mathrm{R}_{\text {Total }}\right)$ and Channel conditions SNR. Total end to end distortion can be minimized by proper selection of source rate and channel rate.

Simulation results comparisons for EEP, UEP and UEP_ED for fixed transmission rate $\left(\mathrm{R}_{\text {Total }}\right)$ and SNR for Barbara image is mentioned in Table 2. Total distortion reduced in UEP_ED algorithm compared to EEP. The results also observed for another values of SNR. The plot of total transmission rate $\left(\mathrm{R}_{\text {Total }}\right)$ versus total distortion for Quality factor $\mathrm{QF}=50$ as shown ion Figure 8 (a). Where total Distortion $\left(\mathrm{D}_{\text {Total }}\right)$ includes source distortion $\left(D_{s}\right)$ and channel distortion $\left(D_{c}\right)$. If channel distortion $\mathrm{D}_{\mathrm{c}}$ value zero than total distortion depends only on quality factor value. So in Figure 8 (b) MSE will be less for higher rate. It is also observed that for lower $\mathrm{SNR}=5$ $\mathrm{dB}$ allocation of higher $\mathrm{QF}$ can also not improve overall distortion.

The comparison of EEP, UEP and UEP_ED algorithms for fix SNR is shown in Figure 8(c). It is observed that approximately $1.5 \mathrm{~dB}$ improvement in PSNR using UEP_ED algorithm compared to UEP in noisy environment. 
Table 2. Comparison of EEP, UEP and UEP_ED at Fix Rate $\left(\mathbf{R}_{\text {Total }}\right)$ for SNR=10dB

\begin{tabular}{|c|c|c|c|c|c|c|c|c|c|}
\hline Image & Method & QF & $\mathbf{R}_{\mathrm{s}}$ & \multicolumn{3}{|c|}{$\boldsymbol{R}_{c}$} & $\mathbf{R}_{\text {Total }}$ & MSE & PSNR \\
\hline \multirow{6}{*}{ Barbara } & EEP & 50 & 1.0381 & \multicolumn{3}{|c|}{$8 / 14$} & 2.14 & 250.406 & 24.144 \\
\hline & & & & $R_{c}^{D C}$ & \multicolumn{2}{|c|}{$R_{c}^{A C}$} & & & \\
\hline & UEP & 50 & 1.0381 & $8 / 16$ & \multicolumn{2}{|c|}{$8 / 13$} & 2.02 & 117.103 & 27.445 \\
\hline & & & & $R_{c}^{D C}$ & $R_{c-H}^{A C}$ & $R_{c-L}^{A C}$ & & & \\
\hline & \multirow{2}{*}{ UEP_ED } & \multirow{2}{*}{50} & \multirow{2}{*}{1.0381} & $8 / 16$ & $8 / 14$ & $8 / 12$ & 2.02 & 81.717 & 29.008 \\
\hline & & & & $8 / 16$ & $8 / 12$ & $8 / 14$ & 203 & 96.827 & 28.271 \\
\hline
\end{tabular}

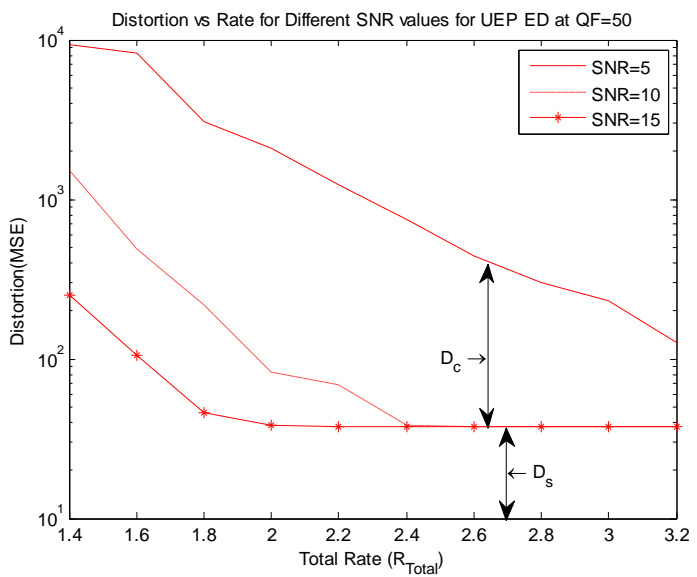

(a)

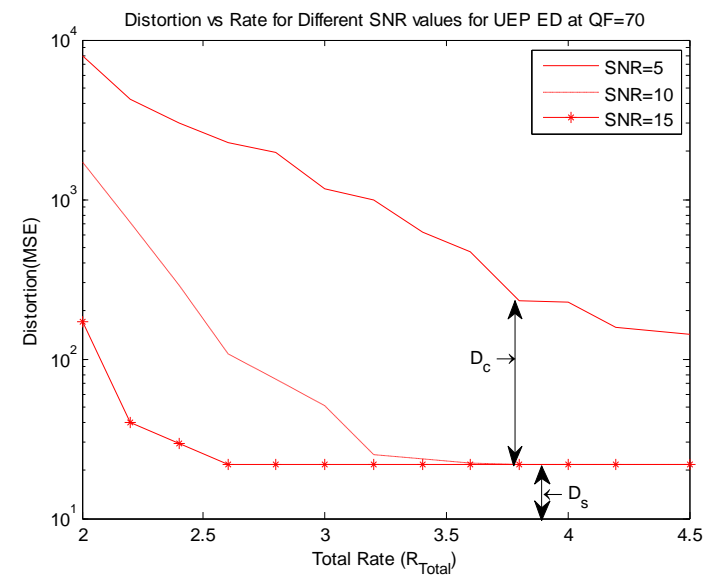

(b)

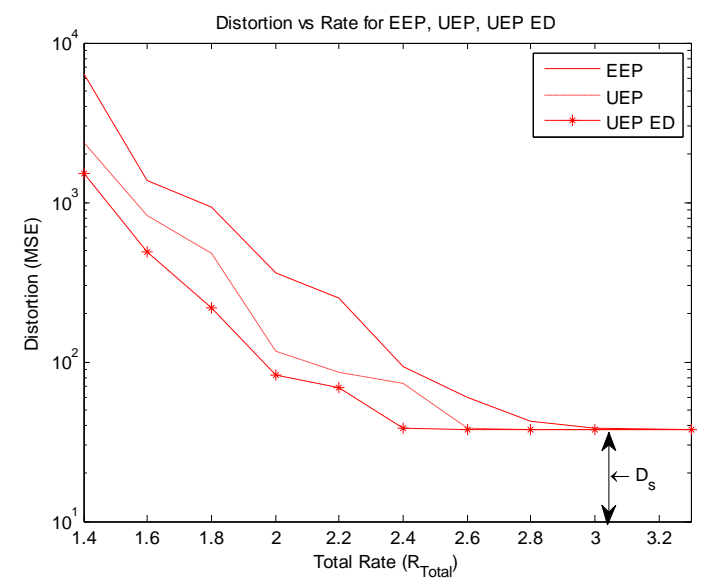

(c)

Fig 8: Total rate versus distortion curve Different SNR for UEP_ED algorithm (a) at $Q F=50$ (b) $Q F=70$ (c) Comparison of EEP, UEP and UEP_ED for fixed SNR

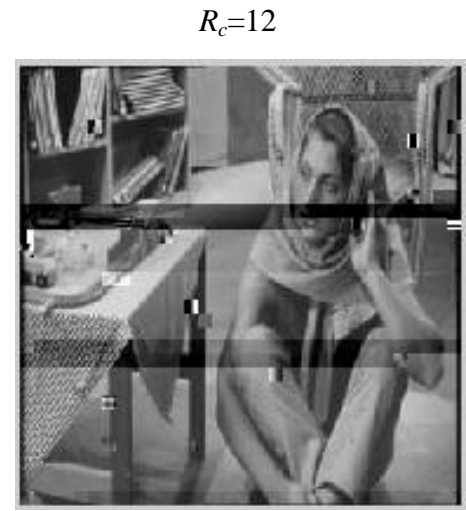

MSE $=936.344$, PSNR $=18.416$

(a)

$$
R_{c}^{D C}=8 / 16, R_{c}^{A C}=8 / 11
$$

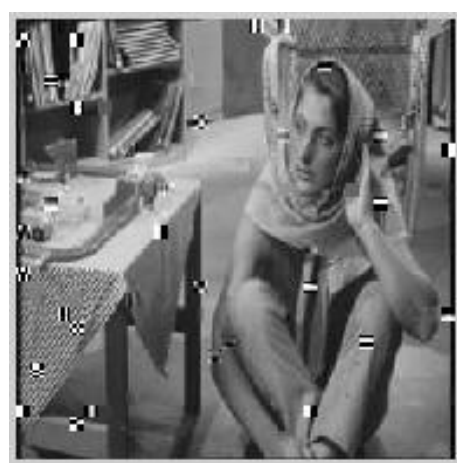

$\mathrm{MSE}=479.050, \mathrm{PSNR}=21.327$

(b)
$R_{c}^{D C}=8 / 15, R_{C-H}^{A C}=8 / 12, R_{C-L}^{A C}=8 / 11$

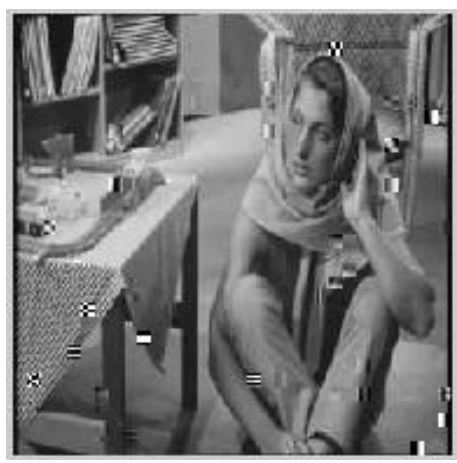

$\mathrm{MSE}=218.094, \mathrm{PSNR}=24.744$

(c)

Figure 9: Decompressed images for (a) EEP (b) UEP and (c) UEP_ED for Barbara, SNR = 10dB 


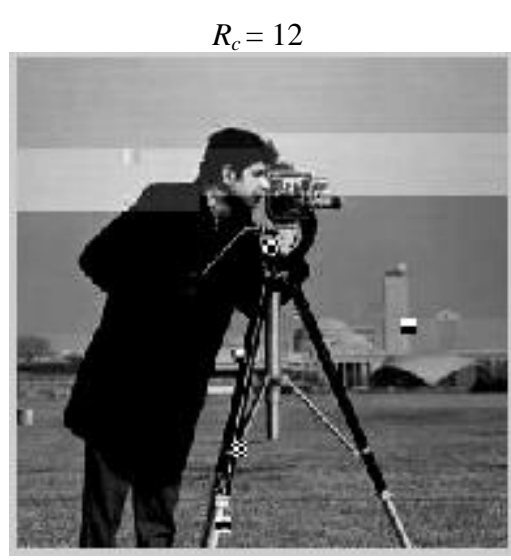

$\mathrm{MSE}=837.123, \mathrm{PSNR}=18.903$

(a)

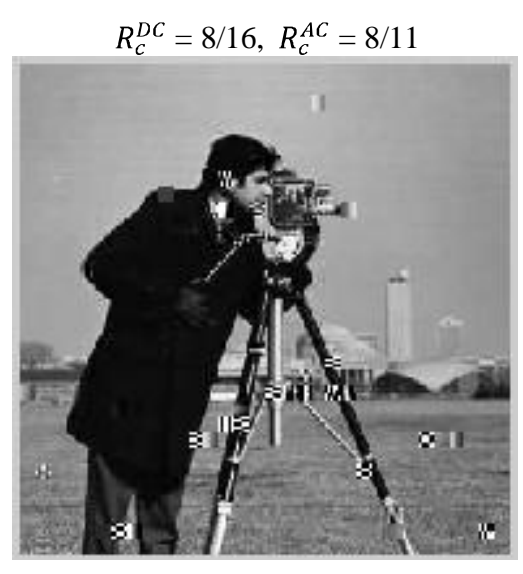

MSE $=365.205$, PSNR $=22.505$

(b)

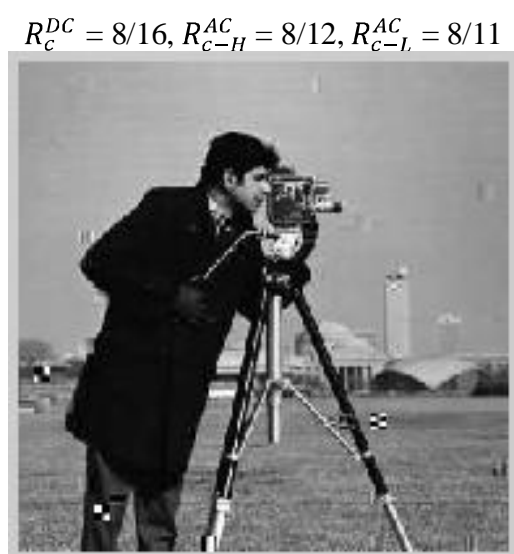

$\mathrm{MSE}=133.371, \mathrm{PSNR}=26.880$

(c)

Figure 10: Decompressed images for (a) EEP, (b) UEP and (c) UEP_ED for Cameraman, SNR=10dB

Figure 9 and 10 shows the visual result of comparison of EEP, UEP and UEP_ED for Barbra image and cameraman image. The visualization quality is improved in UEP_ED algorithm for both the images.

\section{CONCLUSION}

For image, video transmission system Joint Source Channel Coding approach is useful. Using proper data partition and protection level received image quality can be improved. This UEP_ED algorithm can improve the perceptual quality with fixed transmission rate and channel condition. This algorithm can be applied to any types of image like low frequency image or high frequency image. Distortion reduction can be obtained in UEP_ED algorithm at the cost of computation of Edge density. This algorithm can be further extended by data partitioning of image bitstreams with another spatial domain property of block.

\section{REFERENCES}

[1] E.Shanon, "A mathematical Theory of Communication," The Bell system technical journal, vol. 27, pp. 379-423, 1948.

[2] P. G. Sherwood and K. Zeger, "Progressive image coding for noisy channels", IEEE Signal Process. Lett., vol. 4, no. 7, pp. 189-191, Jul,1997.

[3] P. G. Sherwood and K. Zeger, "Error protection for progressive image transmission over memory less and fading channels", IEEE Trans. Commun., vol. 46, no. 12, pp. 1555-1559, Dec. 1998.

[4] A. A. Alatan, M. Zhao, and A. N. Akansu, "Unequal error protection of SPIHT encoded image bit streams", IEEE J. Sel. Areas Commun.,vol. 18, no. 6, pp. 814-818, Jun. 2000.

[5] A. E. Mohr, E. A. Riskin, and R. E. Ladner, "Unequal loss protection: graceful degradation of image quality over packet erasure channels through forward error correction", IEEE J. Sel. Areas Commun., vol. 18, no. 6, pp. 819-828, Jun. 2000.

[6] Z. Wu, A. Bilgin, and M. W. Marcellin, "Unequal error protection for transmission of JPEG2000 codestreams over noisy channels", in Proc. IEEE Int. Conf. Image Processing, Rochester, NY, 2002, pp. 213-216, 2002.

[7] Chou-Chen Wang, Tung-Yuen Huang and chung You Yang, "Joint Source Channel Coding for JPEG Compressed Images over Noisy Channel," Congress on Image and Signal Processing, IEEE Computer society pp. 676-680, 2008.

[8] Gregory K. Wallace, "The JPEG still picture compression standard", Special issue on Digital multimedia systems, Issue 4, vol 34, pp. 30-44, April 1991.

[9] J. Hagenauer, "Rate compatible punctured convolutional codes (RCPC) and their application" IEEE trans. on communication vol. 36 no 4, pp. 389-400, April 1988. 